\title{
Towards Situation-aware Mobile Applications in Mental Health
}

\author{
Ariel S. Teles, Francisco J. Silva \\ Federal University of Maranhão \\ São Luís, Brazil \\ Donal O’Sullivan, Pepijn Van de Ven \\ University of Limerick \\ Limerick, Ireland
}

\author{
Artur Rocha \\ INESC TEC \\ Porto, Portugal
}

\begin{abstract}
This work describes SituMan (Situation Manager), a mobile system that makes use of the sensors commonly included in most mobile platforms and a fuzzy inference engine to attempt to infer user context and environment. Such "situation" information, has been used to enhance the behaviour of MoodBuster, another mobile application used in the scope of the mental health domain to collect Ecological Momentary Assessments (EMA).

EMA has been used in psychotherapy to minimize the effects of recall bias in the assessment of patient mood, as well as in the recollection of other experiences and behaviours. SituMan can enhance the user experience in the scope of EMA by prompting users in the desired situation, instead of at random or fixed-times, thus reducing obtrusiveness. It can also provide new insight to mental health professionals by summarizing the situations experienced by the patient, further allowing correlation of situation information with patient mood within the same time frame.
\end{abstract}

Keywords-Mobile Mental Health; Situation Awareness; Ecological Momentary Assessment;

\section{INTRODUCTION}

Mobile devices provide connectivity and allow accessing, processing and sharing information mostly any time and anywhere. They also incorporate a variety of sensors, which provide the capacity of gathering information about user context and allow the execution of more sophisticated applications - aware of the environment where users are located and identifying their situation at a given moment. Situation awareness in applications allows the correlation of multiple context information, which can be gathered with the aid of a mobile device, to obtain an enhanced understanding of the current user status and environment.

Mobile devices have been applied to the area of mental health as part of medical, psychological, and general health services to aid in the treatment of mental disorders [1]. The use of mobile devices can be effective in the treatment of mental disorders by: (i) providing real-time feedback to patients about behavioural patterns and instructing how to change them, including through adherence to medication; and (ii) enabling reminders, interventions, contacts, and remote consultations, mainly because consultations with patients play a core role in the treatment of mental disorder. Mobile Mental Health encompasses the use of mobile technologies for supporting the adoption and maintenance of a healthy lifestyle, providing remote visualization of mental health conditions, and facilitating interventions such as promoting patient self-care.

Mobile applications focused on a continuous assessment and intervention over the patients' mental status entail some issues:

1) How to accurately assess the patient's mental status minimizing the recall bias and, at the same time, not being excessively intrusive? Recall bias is a systematic error due to differences in accuracy or completeness of recall to memory of past events or experiences. This poses problems in a psychological treatment where questions are made by the psychologist to the patient several days later, because the memory bias alters recalled memories of past events introducing significant differences to what actually happened to the patient. Mobile applications can take advantage of the most appropriate moments to evaluate the patient's mental status in a more precise and unobtrusive way, by requesting timely self-assessments from the patient wherever and whenever the situation is adequate, leading to a context-aware and usercentric triggering of the aforementioned assessments.

2) How to accurately identify situations experienced by the patient in a time period? Having a better insight about the patient's daily routine (with the patient's permission), a psychologist may be able to discuss causes and effects of depressive symptoms (e.g. "are they spending too long at work?"; "are they sleeping for the most part of the day?"), thus improving the effect of therapy. Moreover, the psychologist can correlate situations experienced by patients with the record of their assessments, thus trying to infer which situations have a positive or negative impact over the patient's state of mind. 
In this work SituMan (Situation Manager) is introduced, consisting of a mobile system that addresses the issues of recall bias and situation identification by providing situation awareness to mobile applications such as the ones in the scope of mental health. In SituMan a situation expresses an aspect of the user's everyday live, i.e. it represents daily routine states named by the user, such as "working", "studying", "at mother's house", "socializing", etc. SituMan uses a fuzzy inference engine to infer the user situation from the contextual information specified by the user. In this paper we describe technical aspects of the SituMan development process and its integration with an existing mental health mobile application.

SituMan is composed of an Android mobile application and a shared service with a well-defined Application Programming Interface (API) used in the scope of this work to provide situation awareness to the MoodBuster ${ }^{1}$ [2]. The SituMan mobile application is used 1) to express patient situations of interest for the treatment of depression, 2) to identify when they happen in real-time, 3) to make the handling of situations available to the MoodBuster, and 4) to save all changes of situation in a summary. MoodBuster is a mental health mobile application used to request self-assessments from patients in the context of depression treatment. With the context and situation information provided through the integration of SituMan we expect that participants will be able to respond to MoodBuster questions truly "in the moment". Therefore, by using situation awareness provided by the SituMan, MoodBuster requests for mental status self-assessments are performed in pre-set situations deemed adequate for the question at hand. In addition, SituMan logs patient situations in a summary, allowing further consultation by psychologists or simply helping patients to better recall what happened.

The rest of this paper is organized as follows. Section II gives an overview of the area of situation awareness and mobile mental health, and describes the MoodBuster. Section III describes those research efforts concerned with the use of context-aware mobile systems for contributing in psychological treatments. Section IV presents details of SituMan main implementation issues, highlighting its fuzzy inference engine used for identifying situations. Finally, in Section V conclusions are drawn.

\section{BACKGROUND}

\section{A. Situation Awareness}

Situation is a more powerful piece of contextual information than a single portion of context data, such as location, since it is not rich enough to describe the wide spectrum of possible situations in which a user can be. Therefore, situation awareness is not restricted to the use of isolated pieces of context data, but related to combining the relevant context

\footnotetext{
${ }^{1}$ https://moodbuster.eu
}

data to correctly identify the user situation. Endsley [3] defines situation awareness as the perception of elements in the environment within a volume of time and space, the comprehension of their meaning, and the projection of their status in the near future. Situation identification techniques abstract low level context data into more meaningful high level contexts. According to Ye et al. [4], they can be classified in: (i) specification-based techniques (e.g. fuzzy logic), and (ii) learning-based techniques.

\section{B. Ecological Momentary Assessment \& Intervention}

Ecological Momentary Assessment (EMA) is a mechanism used to prompt individuals, at fixed or random times, to respond to questions about what they are doing (or have done) and/or experiencing (or have experienced), repeatedly, throughout a period of time within their daily routine. According to Shiffman [5], EMA aims "to assess the flow of experience and behaviour over time, capturing life as it is lived, moment to moment, hour to hour, day to day, as a way of faithfully characterizing individuals and of capturing the dynamics of experience and behaviour over time and across settings". Where EMA focusses on obtaining information from the patient as it experienced by the latter, Ecological Momentary Intervention (EMI) "provides a framework for treatments characterized by the delivery of interventions to people as they go about their daily lives" [6].

\section{The MoodBuster Application}

This work aims at complementing MoodBuster, developed in the scope of the ICT4Depression [7] and E-Compared (European Comparative Effectiveness Research on Internetbased Depression Treatment) ${ }^{2}$ projects. E-Compared is conducted by 13 different institutions from the 10 European countries, involving multidisciplinary researchers and professionals from several domains such as psychology, psychiatry, informatics engineering, and computer science. The project goal is to evaluate the impact of blended interventions in the treatment of depression. E-Compared aims to provide mental healthcare stakeholders with evidence-based information and recommendations about the clinical and cost-effectiveness of blended depression treatment. Blended treatment entails a combination of (1) internet and mobilebased, and (2) face-to-face (traditional) assessments and interventions. The central assumption of the project is that the two forms of treatment will lead to similar clinical improvements in patients, but that the blended form can be offered at significantly lower costs, by using information and communication technologies.

The MoodBuster [2] used in ICT4Depression and ECompared projects is an EMA/I mobile application for use in the treatment of depression. A person can use it to get an accurate view of the way that their mood changes over time.

\footnotetext{
${ }^{2}$ http://www.e-compared.eu/
} 
It asks questions about patient's emotional state at various moments of the day and presents accumulated answers in a graph, which can later be consulted by the healthcare professional (e.g. psychologist). This will help their patients to self-assess and identify periods when their mood was lower, eventually allowing better preparation to cope with future events.

\section{RELATED WORK}

Related works in context-aware mobile computing solutions to support health remote monitoring are vast and varied. There is a growing interest in using mobile technology to support treatments of mental disorders [1]. For example, Empath [8] is a remote monitoring system to use in the domestic environment. It combines context information obtained from sensors embedded in mobile device as well as spread in home with patient self-assessments to detect early signs of mental disorders such as depression, in addition to provide information about the effectiveness of treatments. The second version of Empath [9] is designed to be used for different home health care applications. Mobilyze! [10] is another system focused on treatment of depression, which transmits 38 types of context data to a server side that uses machine learning techniques to infer moods, emotions, cognitive/motivational states, activities, environmental contexts, and social interactions of the patient.

MONARCA wearable system [11] aims to recognize the early warning signs of manic and depressive episodes to predict and prevent the occurrence, and to adjust the treatment. This system runs in the mobile device and also in wearable sensors. In addition to provide the communication between patient and the health professional and to receive context information from the wearable devices, the mobile application is used to capture context data from the embedded sensors in mobile device and to estimate other contextual information, such as level of patient's physical and social activity, patient mobility, and mood. In the second version of MONARCA [12] has a mood forecasting component that is used for mood prediction on a 5-day horizon. It is possible to track the patient's daily routine via the MoodRhythm [13] mobile application, which relays this information to clinicians who provide feedback to patients about their routine in order to enable them to improve their mood. MoodRhythm uses context information to estimate the time that the patient spent sleeping as well as their social activity patterns.

BeWell [14] is a mobile application developed to promote improved behavioral patterns to the user via feedback rendered in the smartphone's display. Using context information it monitors user behavior by inferring sleep duration, physical activities and social interactions. Extending BeWell, the StudentLife [15] is used to assesses the day-to-day of a group of students by additionally collecting context data. Its objective is to assess the mental health, academic performance, and behavioral trends of those students.
All these related works deal with patient mobile remote monitoring using contextual information as additional resource for improving and reinforcing psychological treatments and diagnosis. Our proposal differs from them by exploring the use of situation awareness as the basic background for automatic adaptation of requests for selfassessments. In addition, SituMan can register situations experienced by the patient for helping psychologists in their decision making. By providing these functionalities, we argue that the situation-aware MoodBuster leads to a new class of EMA/I mobile application, the so-called Situation-Aware EMA/I (SA-EMA/I), which leads to a more adequate and unobtrusive patient assessment and intervention.

\section{THE PROPOSED MOBILE SYSTEM}

The implemented mobile system comprises a fuzzy inference engine used to identify the patient situations, along with a service to provide situation awareness to the MoodBuster.

\section{A. The Situation Inference Engine}

The following factors influence the design decision of situation inference engine used in SituMan: the application domain, the type and relevance of context information, and the reasoning method used to identify situations. In the scenario of assessment/intervention in mobile mental health, the following requirements led the development of this situation inference solution: (1) patients with help from their psychologists must easily express their situations in the mobile application; and (2) the mobile application must identify the patient's current situation correctly and at the right time.

The situation inference engine described hereby uses the fuzzy logic [16] as basis for identifying situations. We decided to use fuzzy logic mainly for the following reasons:

1) It is widely used to deal with uncertainty of vagueness, having as main objective to model computationally the human reasoning, which is naturally imprecise and qualitative;

2) It provides a notation to represent the inference process using rules that can be easily understood. Fuzzy rules provide a language that uses linguistic variables, which allows the user to express situations that can represent values as terms instead of using crisp sets, and these terms can be represented in friendly user interfaces;

3) It does not require much computing power from current mobile devices to perform the inference process, which avoids a communication with a server or cloud side;

4) There is no need for a training phase, as would be required if supervised machine learning techniques were applied.

In the SituMan a situation is defined as a tuple $S:<$ $L, T, A>$, where $L$ represents the current user location 


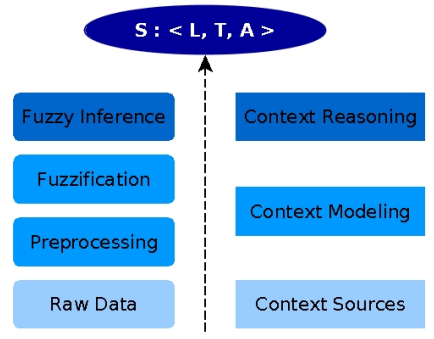

Figure 1. Conceptual Model of User Situation Inference using Fuzzy Inference.

(Where), $T$ is the time represented by "day of the week" and "time on the day" (When), and $A$ represents the current user activity (What). From this definition, a situation is identified from the correlation of the context data using a fuzzy inference engine. Figure 1 shows a conceptual model of the SituMan situation inference process. In this model, context data related to location (L), time (T), and activity (A) are obtained directly from the user mobile device. After collecting the data, which are preprocessed in order to adjust their format, e.g. extract redundant or unused information such as the seconds in a timestamp.

In the fuzzification phase, context information is represented by linguistic variables. Therefore, data about location, time and activity are represented as fuzzy sets: $L=\{$ same place of a user chosen location in a map, near that chosen location, different/distant place than the one chosen by the user $\}, T_{\text {time }}=\{$ dawn, morning, afternoon, night $\}$, $T_{\text {week }}=\{$ weekday, weekend $\}$, and $A=\{$ in vehicle, on bicycle, on foot, running, still, tilting, walking $\}$. Finally, in the fuzzy inference phase, the different types of context information are then combined, correlated and fused to calculate a user situation.

The following example demonstrates how context information can be represented in fuzzy sets. Context information regarding time is expressed using fuzzy sets in a cyclic form: from 0 to 6.99 in Day of Week, where each number represents a day of the week, and from 0 to 23.99 in the case of Time on the Day, where each number represents an hour of the day. As the functions for these sets had to be defined across the limits of a day (from 00:00 to 23:59) and of a week (from Sunday to Saturday) we had to create two sets: "Night1" and "Night2", as well as "Weekend1" and "Weekend2", which overlap and which represent each a single linguistic variable, Night and Weekend, respectively. Therefore, the truth value for weekend is obtained from the $\max ($ Weekend 1, Weekend 2 ), and for night from the $\max (N i g h t 1, N i g h t 2)$. For example, if the user creates a situation using "Night" to express time on the day, the situation inference engine sets the condition of the fuzzy rule to become: if Time on the Day is "Night1" or "Night2". Hence, both sets are considered when checking a situation

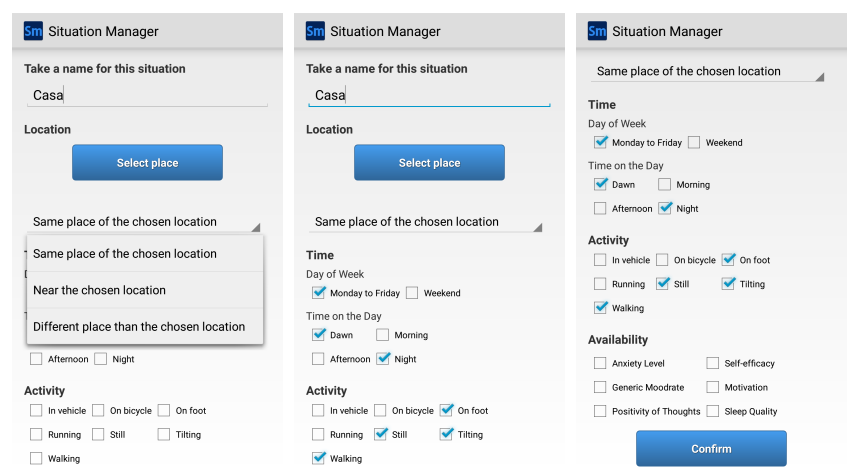

Figure 2. SituMan mobile GUI for defining situations.

fuzzy rule, which guarantees that the rules are correctly evaluated.

\section{B. Definition of Situations}

An essential requirement for adoption of SituMan is the provision of an easy to use interface for the definition of situations. For this, the fuzzy logic provides linguistic variables that facilitate this task. Figure 2 shows the SituMan graphical user interface used to define situations and how it is used to build the situation fuzzy rules that use those variables.

Initially the user defines the name and context information that characterizes the situation, by providing a location, day of week, time on the day, and activity. All context information is required to build a situation. Fuzzy rules that represent situations are created at run time, i.e. SituMan enables the management (addition and removing) of the fuzzy rules (the knowledge base) through the use of the situations definition interface. A knowledge base in a fuzzy system is usually static and created by a specialist in the application field, but in SituMan it is maintained by the patient, and can also have the participation of their psychologist. In this way, the user provides the necessary data to build the fuzzy rules representing their own situations.

Once users have defined their situations of interest, they can choose the types of notifications (i.e. self-assessment requests) that they wish to receive for each situation. The assessments used were: anxiety level, generic moodrate, positivity of thoughts, self-efficacy, motivation, and sleep quality. With this mapping of assessments to situations, SituMan can respond to requests of the MoodBuster application to display a certain assessment. To this end, the MoodBuster application will query the SituMan service API. The invoked method will respond with a message which indicates whether or not the assessment may be presented to the user, given the currently inferred situation.

\section{An Inference Example}

As an example for situation inference, consider the following user defined rule: 


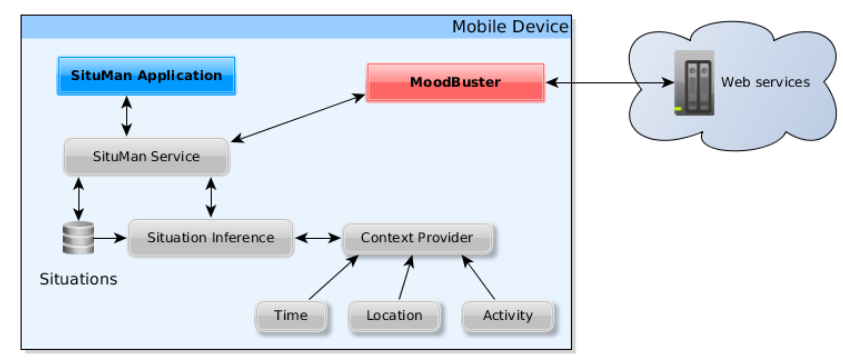

Figure 3. SituMan computational architecture.

if Location = "Same Place" and Day of week = "Weekend" and Time on the day = "Night" and Activity = "still or walking" then the situation = Socializing.

This rule is evaluated by the inference engine as follows:

- Location: the user registers a point in a map and determines the linguistic variable "same place". For this example, we will assume this was a point at their home. At the time of inferencing the engine: (1) checks the current user location, (2) checks the coordinate recorded in the situation (i.e. "user's home"), and (3) then calculates the Euclidean distance between these points to set the input value about this context information;

- Time: the user chooses "Weekend" in the day of week, and checks "Night" in time on the day. At the time of inferencing the engine: (1) checks the current timestamp, (2) format the data to numerical information used in the fuzzy sets regarding time, and (3) sets the input values for this context information;

- Activity: the user specified the activities "Still" and "Walking" in the situation. From this, their probabilities of occurring are summed up and used as input value.

\section{SituMan Architecture}

SituMan runs on the Android platform and is composed of: (i) a shared service that relies on an infrastructure for context data gathering, situation inferencing from gathered data, as well as an API to provide situation awareness to the MoodBuster application in order to request patient self-assessments at the most suitable moments; and (ii) a mobile application (SituMan application) that provides user interfaces to manage situations and visualize a summary of situations experienced by the user. The identified situations that are stored in the summary can be consulted by psychologists in order to learn more about the patient's daily routine and behavioural patterns and, therefore, provide insight for face-to-face sessions during the treatment. The SituMan architecture integrated with the MoodBuster application is illustrated in Figure 3.

The SituMan Application provides interfaces to: (i) configure situations, (ii) check the current user situation, (iii) show and save a $\log$ file of the experienced situations, (iv) search the $\log$ for situations in a time period, i.e. the summary, and (v) guide the user on how to use the App and provide information about the research objectives.

The SituMan Service is an Android service implementing an API to enable situation awareness in other applications. To this end, it defines some basic methods used in both SituMan and MoodBuster applications for: (i) adding and removing situations in the Situations database - addSituation and removeSituation methods, (ii) getting active situations, i.e. those situation fuzzy rules with the degree of activation greater than zero - getActiveSituations method, (iii) getting all situations added - getAllSituations method, (iv) getting the situation summary for a specified time period - getSituationsSummary method, and (v) checking the status (i.e. the availability) for a given type of notification according to the current user situation, i.e. the fuzzy rule with greater degree of activation - checkStatus method. Methods of this API are accessed via AIDL $^{3}$, where clients from different applications can access the service and retrieve non primitive data encapsulated in JSON format.

The Situation Inference component contains the fuzzy inference engine, implemented with the jFuzzyLogic library [17] to detect the current user situation. This component obtains context data from the Context Provider to perform the situation inference. The Context Provider component is responsible for obtaining, formatting and providing context data. Context data about the location are obtained from GPS, mobile networks or Wi-Fi, whereas the local clock of the mobile device provides the time reference. Activity is gathered from the Google Play Services using the activity recognition $\mathrm{API}^{4}$.

\section{CONClusion}

In this paper we propose SituMan, a solution to provide situation awareness to mobile applications, and we describe its integration with the MoodBuster EMA/I mobile application. In this work these two applications address the issues of recall bias and situations identification in the scope of mental health. The MoodBuster application was adapted to use the SituMan Service to address the issues of recall bias and situation identification by providing situation awareness. This service is used by the MoodBuster application to schedule notifications to patients according to their current situation, aiming not be excessively intrusive. These notifications typically request patients to rate their levels of mood, anxiety, sleep quality and rumination. From these situation-aware notifications, the MoodBuster may be able to correlate the patients mental status with their situations. The correlation can be used by the psychologist to identify problematic or good situations experienced by the patients. Moreover, the SituMan Application provides an interface to

\footnotetext{
${ }^{3}$ http://developer.android.com/guide/components/aidl.html

${ }^{4}$ https://developers.google.com/android/reference/com/google/android/ gms/location/ActivityRecognitionApi
} 
search all situations experienced by the patient, an important resource for narrowing the distance between psychologist and patient. This situations summary experienced by the patients can show their daily routine and their behaviour to the psychologist, which is very helpful in the conduct of psychological treatment.

From this work we plan to facilitate the creation of situations by implementing situation templates for the most common ones and to perform further experiments with real patients.

\section{ACKNOWLEDGEMENTS}

The authors would like to thank European Community's Seventh Framework Programme, as well as FAPEMA and CNPQ (Brazilian research funding agencies) for the financial support of this work.

\section{REFERENCES}

[1] F. Gravenhorst, A. Muaremi, J. Bardram, A. Grünerbl, O. Mayora, G. Wurzer, M. Frost, V. Osmani, B. Arnrich, P. Lukowicz, G. Tröster, Mobile phones as medical devices in mental disorder treatment: An overview, Personal Ubiquitous Computing 19 (2) (2015) 335-353.

[2] P. van de Ven, M. R. Henriques, M. Hoogendoorn, M. Klein, E. McGovern, J. Nelson, H. Silva, E. Tousset, A mobile system for treatment of depression, in: International Joint Conference on Biomedical Engineering Systems and Technologies, BIOSTEC'12, 2012.

[3] M. R. Endsley, Toward a theory of situation awareness in dynamic systems, Human Factors: The Journal of the Human Factors and Ergonomics Society 37 (1995) 32-64.

[4] J. Ye, S. Dobson, S. McKeever, Situation identification techniques in pervasive computing: A review, Pervasive and Mobile Computing 8 (1) (2012) 36-66.

[5] S. Shiffman, A. A. Stone, M. R. Hufford, Ecological momentary assessment, Annual Review of Clinical Psychology 4 (1) (2008) 1-32.

[6] K. E. Heron, J. M. Smyth, Ecological momentary interventions: Incorporating mobile technology into psychosocial and health behaviour treatments, British Journal of Health Psychology 15 (1) (2010) 1-39.

[7] A. Rocha, M. Henriques, J. Correia Lopes, R. Camacho, M. Klein, G. Modena, P. Van de Ven, E. McGovern, E. Tousset, T. Gauthier, L. Warmerdam, ICT4Depression: Service oriented architecture applied to the treatment of depression, in: 25th International Symposium on Computer-Based Medical Systems (CBMS 2012), 2012, pp. 1-6.

[8] R. F. Dickerson, E. I. Gorlin, J. A. Stankovic, Empath: A continuous remote emotional health monitoring system for depressive illness, in: Proceedings of the 2nd Conference on Wireless Health, WH'11, ACM, New York, NY, USA, 2011, pp. $1-10$.
[9] R. F. Dickerson, E. Hoque, I. A. Emi, J. A. Stankovic, Empath2: A flexible web and cloud-based home health care monitoring system, in: Proceedings of the 8th ACM International Conference on PErvasive Technologies Related to Assistive Environments, PETRA'15, ACM, New York, NY, USA, 2015, pp. 62:1-62:8.

[10] M. N. Burns, M. Begale, J. Duffecy, D. Gergle, C. J. Karr, E. Giangrande, D. C. Mohr, Harnessing context sensing to develop a mobile intervention for depression, Journal of Medical Internet Research 13 (3) (2011) e55.

[11] A. Puiatti, S. Mudda, S. Giordano, O. Mayora, Smartphonecentred wearable sensors network for monitoring patients with bipolar disorder, in: Engineering in Medicine and Biology Society, EMBC, Annual International Conference of the IEEE, 2011, pp. 3644-3647.

[12] M. Frost, A. Doryab, M. Faurholt-Jepsen, L. V. Kessing, J. E. Bardram, Supporting disease insight through data analysis: Refinements of the monarca self-assessment system, in: Proceedings of the 2013 ACM International Joint Conference on Pervasive and Ubiquitous Computing, UbiComp'13, ACM, New York, NY, USA, 2013, pp. 133-142.

[13] S. Voida, M. Matthews, S. Abdullah, M. C. Xi, M. Green, W. J. Jang, D. Hu, J. Weinrich, P. Patil, M. Rabbi, T. Rahman, G. Gay, E. Frank, T. Choudhury, Moodrhythm: Tracking and supporting daily rhythms, in: Proceedings of the 2013 ACM Conference on Pervasive and Ubiquitous Computing Adjunct Publication, UbiComp'13 Adjunct, ACM, New York, NY, USA, 2013, pp. 67-70.

[14] N. D. Lane, M. Lin, M. Mohammod, X. Yang, H. Lu, G. Cardone, S. Ali, A. Doryab, E. Berke, A. T. Campbell, T. Choudhury, Bewell: Sensing sleep, physical activities and social interactions to promote wellbeing, Journal Mobile Networks and Applications 19 (3) (2014) 345-359.

[15] R. Wang, F. Chen, Z. Chen, T. Li, G. Harari, S. Tignor, X. Zhou, D. Ben-Zeev, A. T. Campbell, Studentlife: Assessing mental health, academic performance and behavioral trends of college students using smartphones, in: Proceedings of the 2014 ACM International Joint Conference on Pervasive and Ubiquitous Computing, UbiComp'14, ACM, New York, NY, USA, 2014, pp. 3-14.

[16] L. A. Zadeh, Fuzzy logic = computing with words, Fuzzy Systems, IEEE Transactions on 4 (2) (1996) 103-111.

[17] P. Cingolani, J. Alcalá-Fdez, jfuzzylogic: a Java library to design fuzzy logic controllers according to the standard for fuzzy control programming, International Journal of Computational Intelligence Systems (2013) 61-75. 